\title{
ARTICLE \\ Inhibition of endocannabinoid degradation rectifies motivational and dopaminergic deficits in the Q175 mouse model of Huntington's disease
}

\author{
Dan P. Covey ${ }^{1}$, Hannah M. Dantrassy ${ }^{1}$, Samantha E. Yohn ${ }^{2,3}$, Alberto Castro ${ }^{1}$, P. Jeffrey Conn ${ }^{2,3,4}$, Yolanda Mateo ${ }^{5}$ and \\ Joseph F. Cheer iD ${ }^{1,6}$
}

Prominent motor deficits (e.g., chorea) that typify Huntington's disease (HD) arise following a prolonged prodromal stage characterized by psychiatric disturbances. Apathy, a disorder of motivation characterized by diminished goal-directed behavior, is one of the earliest and most common psychiatric symptoms in HD, but the underlying neurobiology is unclear and treatment options are limited. Alterations in the endocannabinoid (eCB) and dopamine systems represent prominent pathophysiological markers in HD that-similar to motivational deficits-present early and decline across disease progression. Whether changes in dopamine and $\mathrm{eCB}$ systems are associated with specific behavioral impairments in HD and whether these deficits are amenable to viable treatments is unknown. Here, we show that dopaminergic encoding of effortful drive progressively declines with age in an HD mouse model, and is restored by elevating tissue levels of the eCB 2-arachidonoylglycerol (2-AG) through targeted inhibition of its enzymatic degradation. This work supports aberrant dopaminergic encoding of reward as a neurobiological correlate of apathy in HD, and indicates that cannabinoid receptor-based therapies may benefit neuropsychiatric care for HD.

Neuropsychopharmacology (2018) 43:2056-2063; https://doi.org/10.1038/s41386-018-0107-8

\section{INTRODUCTION}

Huntington's disease (HD) is a heritable, neurodegenerative disorder caused by expansion of CAG (cytosine-adenine-guanine; glutamine) repeats in the huntingtin $(H T T)$ gene $[1,2]$. There is no cure or disease-modifying therapy for HD, and hence treatment is limited to symptom management. The only Food and Drug Administration (FDA)-approved treatment for HD is the dopaminedepleting agent tetrabenazine (TBZ), which is only indicated for the hyperkinetic motor deficits that characterize chorea [3]. Motor dysfunction, however, emerges late in disease progression, typically following the onset of psychiatric impairment by a decade or more $[4,5]$. Apathy, a disorder of motivation characterized by diminished goal-directed behavior, is one of the most common and debilitating psychiatric symptoms in HD [5-7]. Motivational deficits are an inherent feature of HD pathogenesis, presenting early in the prodromal stage and worsening throughout disease progression [7]. However, the underlying neurobiology remains unclear and effective therapies are therefore lacking.

Striatal medium spiny neuron degeneration represents the canonical, histopathological hallmark of $\operatorname{HD}[8,9]$. However, before gross neurodegeneration and motor symptoms present, alterations in neurochemical transmission arise during periods when patients primarily exhibit psychiatric impairments [10]. Disruptions in endocannabinoid (eCB) and dopamine system function are prominent neuropathologies in HD that present early and worsen across disease progression [11-13]. Deficits occur in dopamine [14] and cannabinoid type 1 (CB1) [15] receptor expression and function as well as in brain levels of dopamine [16] and the endogenous $C B 1$ receptor ligand 2-arachidonoylglycerol (2-AG) [17]. Because 2-AG signaling at CB1 receptors regulates dopamine neurotransmission and goal-directed motivation [18, 19], dysfunction in these neuronal systems may contribute to apathy in HD. However, whether progressive changes in dopamine function associate with specific behavioral symptoms characteristic of apathy in $\mathrm{HD}$ and whether $\mathrm{eCB}$ modulation of dopamine function influences these impairments are unclear.

We recently observed a motivational deficit coincident with compromised dopaminergic input to the nucleus accumbens (NAc) of adult (10 months old) Q175 mice [20]. This knock-in HD mouse model expresses the human $\mathrm{Htt}$ allele containing expanded CAG repeats ( 179) within the native mouse $H T T$ gene [21]. The apathetic phenotype is characterized by diminished motivation to work for a reward and associated with reduced dopamine release in the NAc following reward receipt, only when effortful costs are high. Because deficits emerge prior to gross motor dysfunction, we consider these measures as a model of prodromal HD. However, whether compromised dopamine dynamics and suppressed effortful responding in this HD model characterize a valid endophenotype of HD or if they are

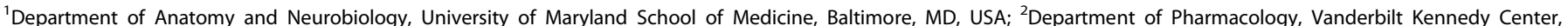

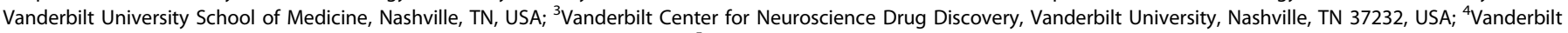

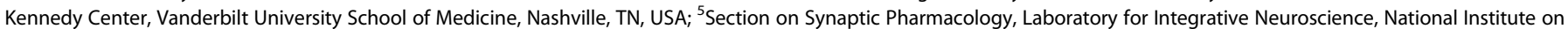

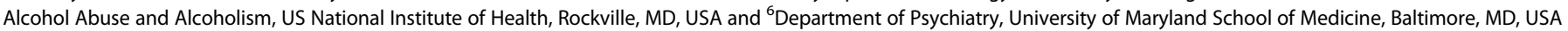
Correspondence: Joseph F. Cheer (jcheer@som.umaryland.edu)

Received: 13 April 2018 Revised: 18 May 2018 Accepted: 24 May 2018

Published online: 1 June 2018 
epiphenomena unrelated to symptom progression is unclear. Additionally, whether deficits can be rectified through a clinically viable strategy is unknown. Here we show that this motivational deficit manifests as dopamine function progressively declines across the lifespan, as occurs in HD, and pharmacological elevation of 2-AG signaling at $\mathrm{CB} 1$ receptors normalizes behavioral and dopaminergic impairments. This work supports aberrant dopaminergic encoding of reward as a neurobiological correlate of apathy in $\mathrm{HD}$, and indicates that cannabinoid receptor-based therapies may benefit neuropsychiatric care for HD patients.

\section{MATERIALS AND METHODS}

Animals

All mice $(20-30 \mathrm{~g})$ were male and received from the Jackson Laboratories (Bar Harbor, ME) at 10 or 30 weeks of age. Mice were housed in a temperature-controlled room maintained on a reverse $12 \mathrm{~h}$ light/dark cycle (0700-1900h). Following surgery, mice were housed individually and allowed ad libitum access to water and food. Mice were restricted to $85-90 \%$ bodyweight for the duration of behavioral measures. Of note, wild-type (WT) and HD mice are of similar bodyweights, as we have previously shown [20]. All experiments were conducted in the animal's light cycle. Animal care and experimental procedures conformed to the National Institutes of Health Guide for the Care and Use of Laboratory Animals and were approved by the Institutional Animal Use and Care Committee at the University of Maryland, Baltimore.
Surgery

Mice were anesthetized with isoflurane in $\mathrm{O}_{2}(4 \%$ induction and $1 \%$ maintenance) and implanted with a chronic voltammetry electrode targeting the NAc core (+1.2 anteroposterior (AP), +1.1 mediolateral (ML), -3.7 dorsoventral (DV) relative to Bregma) and a $\mathrm{Ag} / \mathrm{AgCl}$ reference electrode in the contralateral superficial cortex. All components were permanently affixed with dental cement (Grip Cement Dentsply).

In vivo fast-scan cyclic voltammetry

Fast-scan cyclic voltammetry (FSCV) at chronically implantable electrodes [22] was used to monitor dopamine concentration changes during the progressive-ratio (PR) task as previously described [20]. Individual carbon fibers $(r=3.5 \mu \mathrm{m}$, Hexcel Corporation) were aspirated into a $5 \mathrm{~mm}$ length segment of fused silica. The seal between the silica and carbon fiber was created by applying a two-part epoxy (Super Glue Corporation; TQs12 Epoxy). The exposed carbon fiber was then cut to $\sim 150 \mu \mathrm{m}$ and a silver connector was attached on the opposite end for conductivity. A triangular waveform $(-0.4 \mathrm{~V}$ to $+1.3 \mathrm{~V}$ at $400 \mathrm{~V} / \mathrm{s})$ was applied at $10 \mathrm{~Hz}$ to implanted carbon fiber microelectrodes. Principal component regression (PCR) was used to statistically extract the dopamine component from the voltammetric recording of current $[23,24]$. The training set for PCR consisted of five backgroundsubtracted dopamine, and five basic $\mathrm{pH}$ shift voltammograms. Measured current was converted to concentration based on a data set developed in vitro using a flow cell apparatus to quantify dopamine oxidation current versus non-faradaic background current [25]. The reward-evoked dopamine signal was quantified a
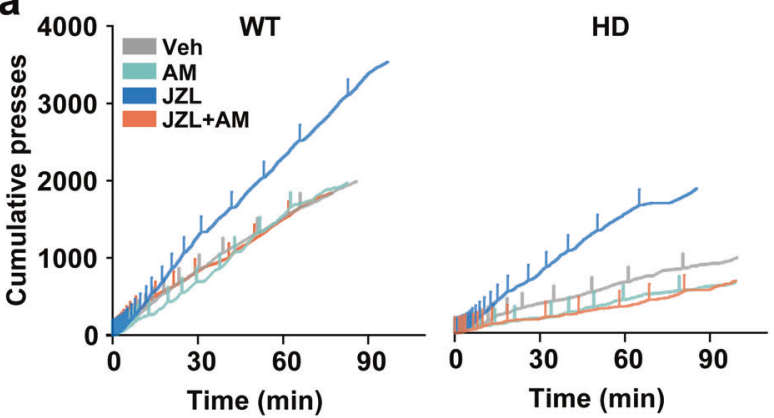

C

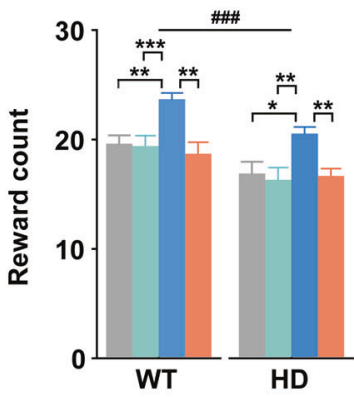

d

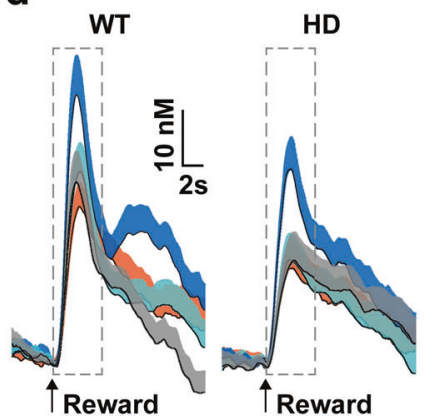

b
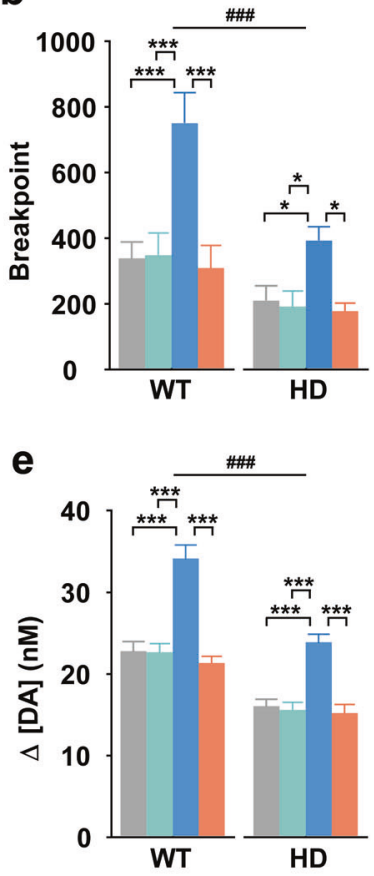

Fig. 1 Motivation and dopaminergic encoding of reward is compromised in 10-month-old HD mice and facilitated by endocannabinoids. a Representative cumulative responding during the progressive ratio task for a sucrose pellet reward in WT (left panel) and HD (right panel) mice following each drug treatment. Treatments are vehicle (VEH: WT, $n=11 ; \mathrm{HD}, n=9$ ), AM-251 (AM: WT, $n=10 ; \mathrm{HD}, n=9$ ), JZL-184 (JZL: WT, $n=10 ; \mathrm{HD}, n=11$ ), and JZL-184+AM-251 (JZL+AM: WT, $n=10 ; \mathrm{HD}, n=9$ ). Vertical tick marks demarcate reward receipt. Drug treatment increased $\mathbf{b}$ mean (+SEM) breakpoints (main effect treatment; two-way ANOVA: $F_{(3,71)}=16.32, p<0.001$ ) and $\mathbf{c}$ rewards earned (main effect treatment; two-way ANOVA: $\left.F_{(3,71)}=12.74, p<0.001\right)$ during PR sessions, which were both greater in WT versus HD mice (Breakpoint; main effect genotype; two-way ANOVA: $F_{(1,71)}=34.63, \# \# p<0.001$; Reward count; main effect genotype; two-way ANOVA: $F_{(3.71)}=29.44$, \#\#\# $p<$ $0.001)$. d Mean (+SEM) dopamine concentrations (DA) aligned to reward receipt (arrow). Drug treatment increased e mean (+SEM) change $(\Delta)$ in reward-evoked (DA) (main effect treatment; two-way ANOVA: $F_{(3,71)}=28.89, p<0.001$ ), which was greater in WT versus HD mice (main effect genotype; two-way ANOVA: $\left.F_{(3.71)}=84.13,{ }^{\# \# \#} p<0.001\right)$. Time period during which [DA] was compared is demarcated by vertical dashed lines in (d). Post hoc $t$-test; ${ }^{* * *} p<0.001,{ }^{* *} p<0.01,{ }^{*} p<0.05 \mathrm{JZL}$ versus other treatments 
as the maximal change in dopamine concentration during the $5 \mathrm{~s}$ period following reward delivery (see dashed lines in Fig. 1d) relative to $0.5 \mathrm{~s}$ preceding reward delivery. Voltammetry data from a recording session were included in the analysis if all rewardevoked dopamine measures met the statistical criteria for the PCR analysis.

\section{In vitro fast-scan cyclic voltammetry}

Coronal slices including NAC were prepared from 10-month-old WT and HD mice following methods described previously [26]. Slices were placed in an interface chamber and continually perfused $(2 \mathrm{ml} / \mathrm{min})$ with artificial cerebrospinal fluid (ACSF) containing (in $\mathrm{mM}$ ): $\mathrm{NaCl} 126, \mathrm{KCl} 2.5, \mathrm{NaH}_{2} \mathrm{PO}_{4} 1.2, \mathrm{CaCl}_{2}$ 2.4, $\mathrm{MgCl}_{2}$ 1.2, $\mathrm{NaHCO}_{3} 25$, Glucose 11, HEPES 20, L-ascorbic acid 0.4, $\mathrm{pH} 7.4$, temperature $32^{\circ} \mathrm{C}$. FSCV recordings of electrically evoked dopamine release were performed using Demon acquisition and analysis software [27] at a glass-encased cylindrical carbon fiber placed in the NAc. Stimulation pulses ( $2 \mathrm{~ms}$ pulse width) were delivered by a constant current isolated stimulator (A-M Systems, WA) through a bipolar tungsten electrode in contact with the slice. Stimulation pulse duration and timing were controlled by a Master-8 (A.M.P.I., Jerusalem, Israel). Dopamine release was monitored using FSCV by applying a triangular waveform $(-0.4$ to $+1.2 \mathrm{~V}$ at $400 \mathrm{~V} / \mathrm{s}$ ) at $10 \mathrm{~Hz}$ to the carbon fiber. An input-output response curve was generated by applying increasing stimulation intensities $(100-600 \mu \mathrm{A})$. Rate of dopamine uptake was determined from the $0.4 \mathrm{~mA}$ evoked signal using a first-order rate constant $(k)$ with Demon Analysis [27]. For pharmacological assays, stimulation was controlled using Axon pClamp 9 Electrophysiological Data Acquisition and Analysis. Drugs were applied to the bath as vehicle (VEH), followed by JZL-184 (1 or $2 \mu \mathrm{M})$, and then sulpiride $(2 \mu \mathrm{M})$. Following the onset of drug application, electrical stimulation $(0.3 \mathrm{~mA})$ was applied to the slice every $2.5 \mathrm{~min}$. The evoked dopamine concentration was averaged across 3 stimulations and then compared across groups and treatments.

\section{Apparatus}

Mice were tested in operant chambers $(21.6 \mathrm{~cm} \times 17.6 \mathrm{~cm} \times 14 \mathrm{~cm}$; Med Associates, St Albans, VT) housed within sound-attenuating enclosures. Each chamber was equipped with two retractable levers (located $2 \mathrm{~cm}$ above the floor) and one LED stimulus light located above each lever $(4.6 \mathrm{~cm}$ above the lever). An external food magazine delivered sucrose pellets ( $14 \mathrm{mg}$; Bio-Serv, Frenchtown, NJ) to a dispenser centrally located between the two levers. A house light and a white-noise speaker $(80 \mathrm{~dB}$, masking noise background) were located on the opposite wall.

\section{Behavior}

Mice were trained to lever press for sucrose pellet reinforcement as previously described [20]. Training began with 30 min sessions of a fixed-ratio (FR) 1 schedule that included a $10 \mathrm{~s}$ timeout following reward receipt which WT and HD mice perform at similar levels [20]. Mice were switched to PR after stable responding was established (under 15\% variation in response rate across three consecutive sessions). The PR schedule of positive reinforcement was used to quantify the effort that animals were willing to expend for a reward [28]. The number of lever presses required to earn each reward (i.e., response ratio) increased exponentially across successive trials (response ratio $=$ $\left.\left[5 \times \mathrm{e}^{(0.2 \times \text { reward number })}-5\right]\right)$, yielding ratios of $1,2,4,6,9,12,15,20$, $25,32,40,50,62,77,95,118$, etc. after rounding to the closest integer. The final ratio attained (i.e., breakpoint) is considered a metric of inherent motivation to expel effort to gain reward. FSCV recordings and drug treatments took place after individual animals displayed stable responding on the PR task (varying by no more than \pm 1 response ratio over 3 consecutive sessions). Session onset was signaled by both levers extending and illumination of the house light and cue light above the active lever. Responses on the inactive lever were recorded but had no programmed consequences. Upon reaching the response requirement on each trial, a single pellet was delivered, both levers were retracted, and house and cue lights dimmed for a $20 \mathrm{~s}$ period before the next trial began. Sessions were terminated after $20 \mathrm{~min}$ passed without reward delivery.

\section{Pharmacology}

Mice were treated prior to the PR session with vehicle, AM-251 $(0.75 \mathrm{mg} / \mathrm{kg}), \quad J Z L-184(18 \mathrm{mg} / \mathrm{kg})$, or JZL $(18 \mathrm{mg} / \mathrm{kg})+A M-251$ $(0.75 \mathrm{mg} / \mathrm{kg})$. Injections were administered intraperitoneally and assigned using a Latin square design with a minimum of 3 days between treatments $[29,30]$. Pretreatment times were 30 min for AM-251 and vehicle, and $120 \mathrm{~min}$ for JZL [31]. Drugs were prepared in a 1:1:18 vehicle consisting of emulphor, ethanol, and saline, respectively. For slice voltammetry recordings, JZL-184 was dissolved in the VEH solution consisting of $0.1 \%$ dimethyl sulfoxide and $1.5 \%$ cyclodextrin dissolved in ACSF. Sulpiride was dissolved in ACSF.

\section{Statistics}

Mean dopamine concentration changes and behavioral measures were compared between genotypes and across drug treatments using a two-way analysis of variance (ANOVA). Because drug treatments similarly affected both genotypes (i.e., no genotype $x$ treatment interaction) and we were primarily interested in treatment effects, subsequent drug treatment effects were compared separately within each genotype using two-way repeated measures ANOVAs. Dopamine release in brain slices was compared across groups and stimulation parameters or drug treatments using a two-way repeated measures ANOVA. Holm-Sidak post-hoc tests were used when both main effects or the interaction was significant. Comparisons between two groups were made using two-tailed Student's $t$-tests. Correlations were statistically compared using a one-way ANOVA after transforming Pearson's correlation coefficients $(r)$ to the normally distributed variable $z^{\prime}$ using Fisher's $z^{\prime}$ transformation. Statistical tests were performed with SigmaPlot (version 12.5) and significance was set at $p<0.05$.

\section{RESULTS}

We assessed whether increasing levels of the eCB 2-AG rectifies motivational deficits in HD mice [20] by systemically inhibiting 2AG degradation in mice performing a PR task for a sucrose reinforcer. Elevating tissue levels of 2-AG with the monoacylglycerol lipase (MAGL) inhibitor JZL-184 $(18 \mathrm{mg} / \mathrm{kg}$, intraperitoneally (i.p.)) [31] increases the motivation to overcome response costs during the PR session in both WT and HD mice, and this effect is blocked by co-administration of a dose of the CB1 receptor antagonist AM-251 (0.75 mg/kg, i.p.; Fig. 1a-c) that has no effect alone, demonstrating CB1 receptor dependence. Behavior is similar within each genotype prior to drug treatment (Supplemental Fig. 1) and drug treatments similarly affect both groups, despite inherently lower overall responding in HD mice, who work less (Fig. 1b) to earn fewer rewards (Fig. 1c).

Dopamine released in the NAc controls motivation to obtain rewards [32] and deficient NAc dopamine release accompanies suppressed effortful responding in adult (10 months old) HD mice [20]. To assess whether eCB signaling controls dopaminergic encoding of reward costs, we used FSCV to monitor subsecond dopamine released in the NAc to reward receipt during the PR task. Drug effects on dopamine release are similar to those on PR responding, such that JZL-184 increases the concentration of dopamine released to reward receipt in both groups and this is blocked by a subthreshold dose of AM-251, despite lower overall levels of responding in HD mice (Fig. 1d, e). Notably, HD mice become indistinguishable from WT controls following JZL-184 
treatment (Supplemental Fig. 2). Thus, dopaminergic encoding of high reward cost is deficient in HD mice and restored to levels observed in WT mice by increasing 2-AG signaling at CB1 receptors.

Whether augmenting 2-AG signaling in HD mice indiscriminately potentiates dopamine released in the NAC or restores information encoded by dopamine neurons is not clear. To address this question, we compared dopamine release across the PR session, as effortful (i.e., lever presses) and temporal (i.e., time to earn reward) demand exponentially increases on successive trials. Dopamine release increases across the PR session in WT mice in all treatment groups, reaching the highest levels late in the PR session, and this effect is potentiated by JZL-184 in a CB1 receptor-dependent manner (Supplemental Figs. 3 and 4).
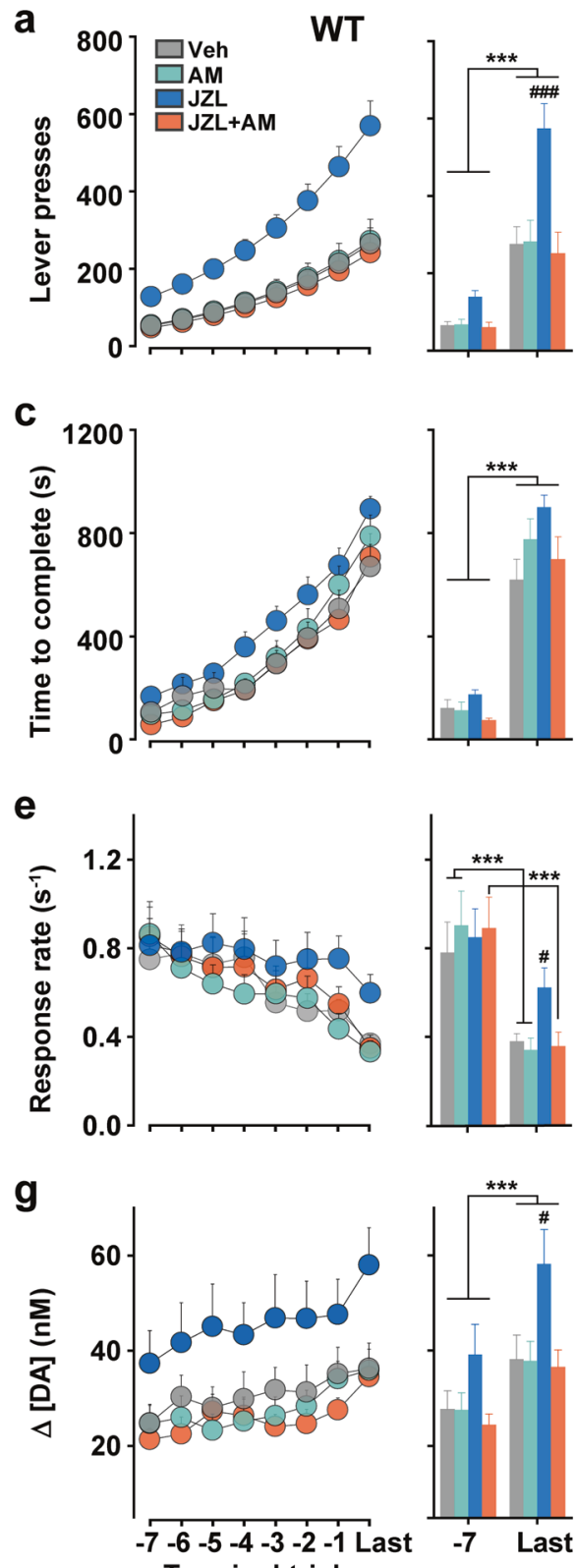

b
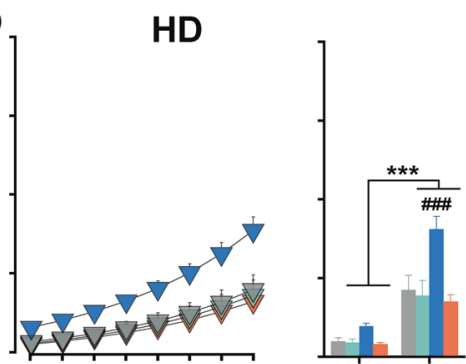

d

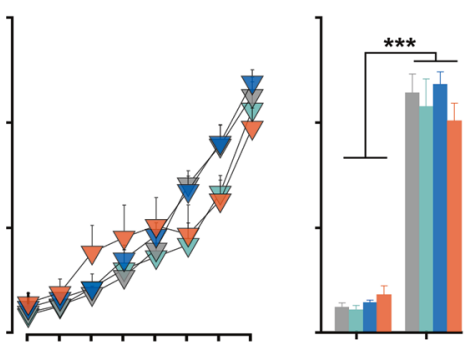

f

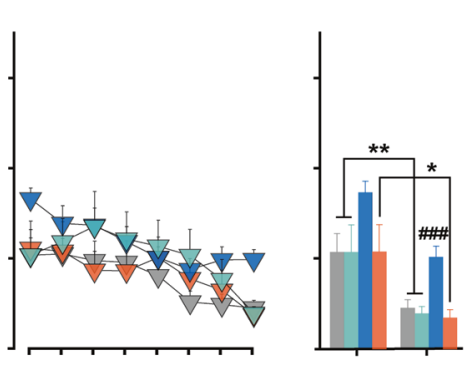

h

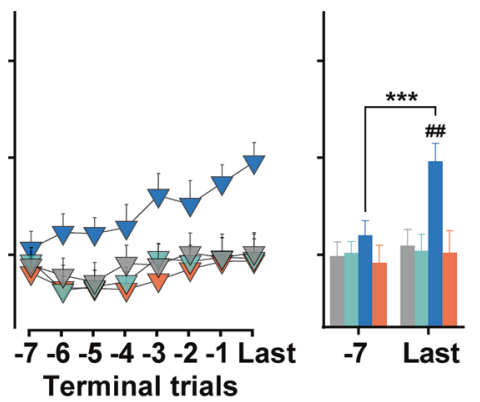

Fig. 2 JZL increases effortful responding and dopaminergic encoding of reward during the final trials of the PR session. Drug treatment increased mean ( + SEM) number of lever presses per reward in a WT (two-way RM ANOVA: drug $\times$ time interaction; $\left.F_{(3,83)}=8.31, p<0.001\right)$ and b HD (two-way RM ANOVA: drug $\times$ time interaction; $\left.\left.F_{(3,79}\right)=8.02, p<0.001\right)$ mice across the final 8 trials ( $x$-axis) of each animal's PR session. Mean (+SEM) time to complete each trial increased in c WT (two-way RM ANOVA: main effect time; $\left.F_{(1,83)}=373.49, p<0.001\right)$ and $\mathbf{d}$ HD (twoway RM ANOVA: main effect time; $\left.F_{(1,79)}=536.74, p<0.001\right)$ mice as the session progressed, but this was not altered by drug treatment (WT: two-way RM ANOVA; main effect treatment; $F_{(3,83)}=2.42, p=0.08$; HD: two-way RM ANOVA; main effect treatment; $\left.F_{(3,79)}=0.47, p=0.71\right)$. Mean $\left(+\right.$ SEM) response rate (lever presses $\times \mathrm{s}^{-1}$ ) decreased across terminal trials in both e WT mice (two-way RM ANOVA: drug $\times$ time interaction; $\left.F_{(3,83)}=3.16, p=0.04\right)$ and $\mathbf{f} \mathrm{HD}$ mice (two-way RM ANOVA: main effect time; $F_{(1,79)}=17.93, p<0.001$, main effect treatment; $F_{(3,79)}$ $=13.09, p<0.001)$, and this decrease was attenuated by JZL. Drug treatment increased mean $(+$ SEM) change $(\Delta)$ in $(D A)$ following reward receipt as the session progressed in g WT (two-way RM ANOVA: main effect treatment; $F_{(3,83)}=3.38, p=0.02 ;$ main effect time; $F_{(1,83)}=83.34$, $p<0.001$ ) and $\mathbf{h}$ HD (two-way RM ANOVA: drug $\times$ time interaction; $F_{(3,79)}=7.68, p<0.001$ ) mice. While (DA) increased in WT mice in all treatment groups, (DA) only increased in HD mice following JZL treatment. Post-hoc t-test, ${ }^{* * *} p<0.001,{ }^{* *} p<0.01,{ }^{*} p<0.05, '-7$ ' versus 'Last'; ${ }_{\# \# \# p} p<0.001,{ }^{\# \#} p<0.01, \# p<0.05 \mathrm{JZL}$ versus treatments within 'Last' 
Moreover, dynamic dopaminergic encoding of increasing costs only occurs in HD mice following treatment with JZL-184promoting a similar pattern as observed in WT mice-and this effect is blocked by AM-251 (Supplemental Figs. 3 and 4).

To specifically assess how dopamine release associates with an animal's willingness to work for a reward, we analyzed terminal trials of the PR session as mice reach their respective breakpoint (Supplemental fig. 5; Fig. 2). Treatment with JZL-184 increases lever presses in both WT and HD groups (Fig. 2a, b)-as expected from the increased breakpoint-but does not alter the time to earn reward, which did not differ across treatments in either group (Fig. 2c, d). This differential effect is driven by JZL-184 reducing the decay in response rate (i.e., lever presses per second) as reward cost increases across terminal trials, which occurs in both groups following all other treatments (Fig. 2e, f). Moreover, as WT mice reach their respective breakpoints, reward-evoked dopamine release increases in all treatment groups and is potentiated by JZL-184 (Fig. 2g), but dopamine release only increases in HD mice following JZL-184 treatment (Fig. 2h). Importantly, all effects of JZL-184 are blocked by AM-251. As observed for each individual metric, JZL-184 altered the association between behavior and dopamine measures across terminal trials such that HD mice more closely resemble WT controls (Supplemental fig. 6). Thus, increasing 2-AG signaling at $C B 1$ receptors restores deficient effortful drive and dopaminergic encoding of increasing reward costs in HD mice.

Because $\mathrm{HD}$ is a progressive neurodegenerative disorder, we assessed whether the behavioral and dopaminergic deficits observed in adult HD mice (10 months old) manifest as they age, as would be expected in a valid HD model. We find that neither behavior nor dopamine measures differ between groups at 3 months of age (Fig. 3). Specifically, both groups exhibit similar breakpoints (Fig. 3a), earn a similar number of rewards (Fig. 3b), and display similar dopaminergic encoding of reward receipt (Fig. 3c) throughout the PR session, and during terminal trials (Fig. 3d-f).

As prior work demonstrates compromised exocytotic dopamine release in the more aggressive R6/2 mouse HD model [33], we assessed whether a similar deficit explains deficient dopaminergic encoding of reward in 10-month-old HD mice by recording dopamine released by electrical stimulation in NAc brain slices (Fig. 4). The concentration of dopamine released during stimulation is reflective of dopamine vesicle content and release probability, while the decaying portion following stimulation represents transporter-mediated dopamine uptake [26]. Dopamine evoked by a single stimulus pulse similarly increases in WT and HD mice as stimulation intensity increases (Fig. 4a, b) and during 'phasic' stimulation trains (Fig. 4c), demonstrating intact vesicular dopamine function in Q175 HD mice. Moreover, we found no difference in signal decay following stimulation (Fig. 4d). Thus, compromised dopamine dynamics in 10-month-old HD mice are not explained by altered dopamine terminal function in the NAc. We further assessed whether JZL-184 alters dopamine release in brain slices via local actions at NAc terminals, and found no effect of JZL-184 on dopamine released by a single electrical pulse or by a stimulation train (Supplemental fig. 7). Collectively, our findings indicate that deficient reward-evoked dopamine release in 10-month-old HD mice and the ability of JZL184 to restore this deficit do not arise from alterations in terminal dopamine function, but are more likely explained by differences in midbrain dopamine cell firing.
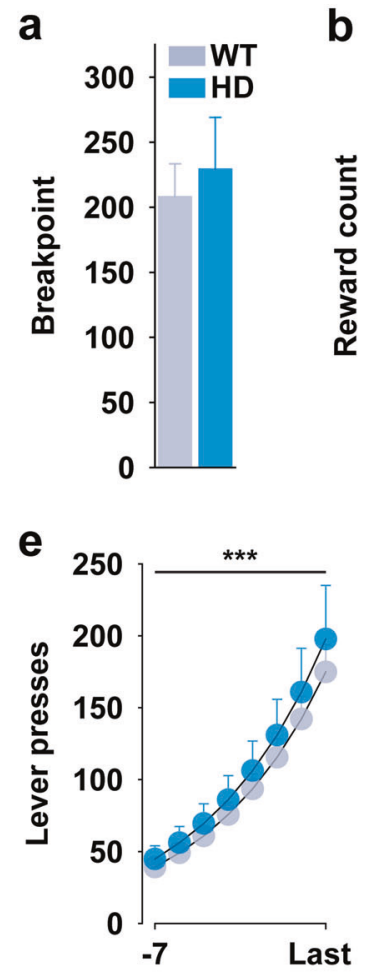

Terminal trials

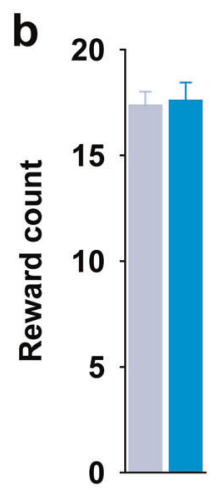

C

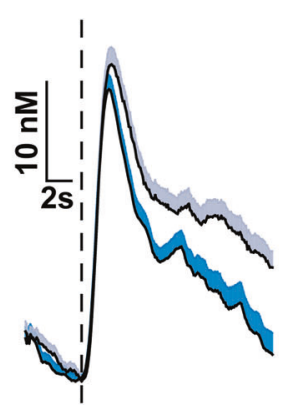

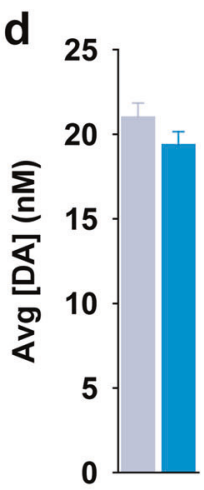

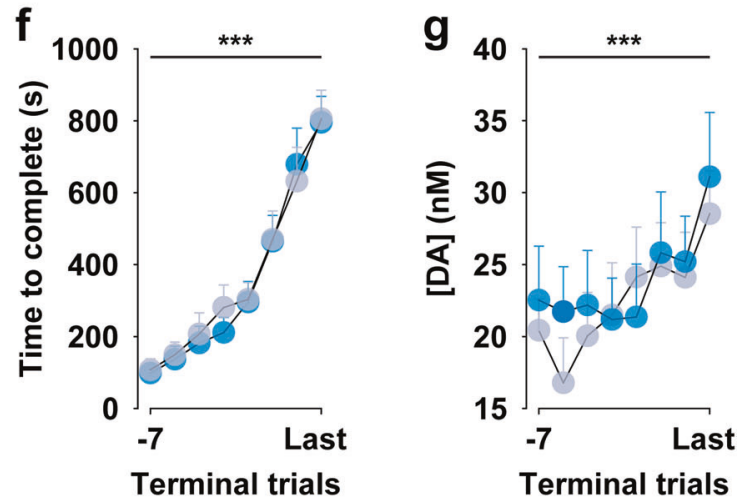

Fig. 3 Intact motivation and dopaminergic encoding of reward in 3-month-old Q175 $(n=11)$ versus WT $(n=11)$ mice. No difference in a breakpoints $(p=0.20)$, $\mathbf{b}$ rewards earned $(p=0.28)$, or $\mathbf{c}$, $\mathbf{d}$ reward-evoked dopamine concentrations (vertical dashed line demarcates reward delivery; $p=0.31)$. No difference between WT and HD mice in e number of lever presses (two-way RM ANOVA: main effect group; $F_{(1,175)}=$ $0.862, p=0.364)$, $\mathbf{f}$ time to complete each trial (two-way RM ANOVA: main effect group; $F_{(1,175)}=0.031, p=0.863$ ), and $\mathbf{g}$ reward-evoked (DA) (two-way RM ANOVA: main effect group; $\left.F_{(1,175)}=0.0007, p=0.979\right)$, all of which increased during the final trials of each animal's PR session (two-way RM ANOVA, main effect of trial, all $\left.{ }^{* * *} p<0.001\right)$. Data are shown as mean (+SEM) 
a

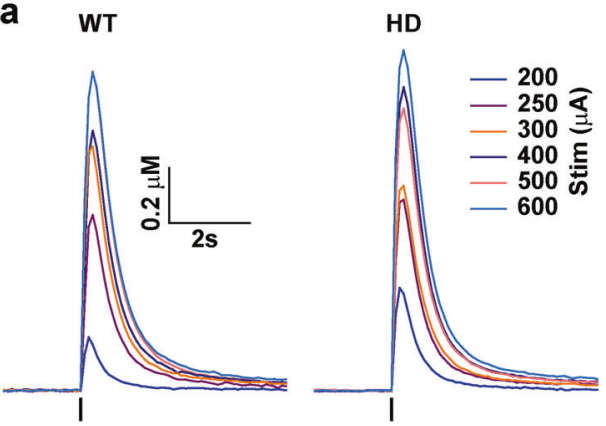

b

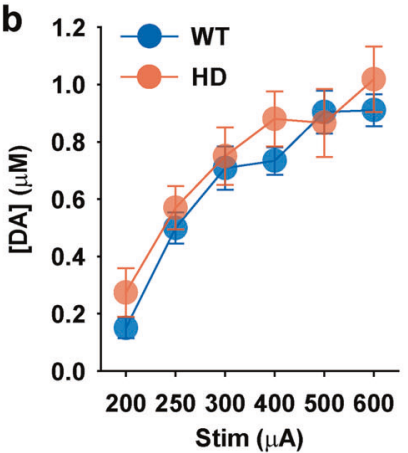

C

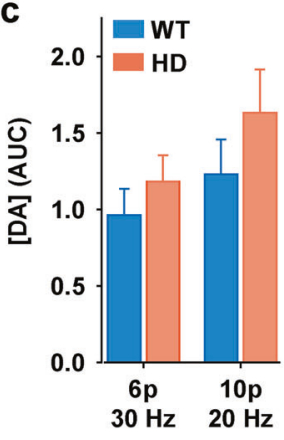

d

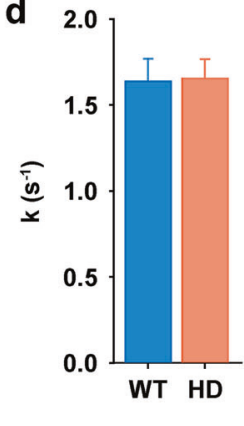

Fig. 4 No difference in terminal dopamine release and uptake at 10 months. a Average single pulse, electrically evoked dopamine release in brain slices from WT (left, $n=10)$ and HD (right, $n=13)$ mice increases according to stimulation intensity $($ Stim, $\mu \mathrm{A})$. b Average $( \pm \mathrm{SEM})$ dopamine release at increasing stimulation intensities (two-way RM ANOVA: main effect group; $F_{(1,129)}=2.38, p=0.137$; main effect stim; $F_{(5,129)}=84.77, p<0.001$; group $\times$ stim interaction; $\left.F_{(5,129)}=0.86, p=0.511\right)$. c Average $( \pm \mathrm{SEM})$ dopamine released (area under the curve $(\mathrm{AUC})$ ) during stimulation trains (two-way RM ANOVA: main effect group; $F_{(1,42)}=2.03, p=0.163$; main effect stim; $F_{(5,42)}=2.68, p=0.11 ;$ group $\times$ stim interaction; $\left.F_{(5,42)}=0.17, p=0.680\right)$. d Average $( \pm S E M)$ dopamine uptake (first order rate constant, $\mathrm{k}$ ) following a single electrical pulse $(400 \mu \mathrm{A})$ $\left(t\right.$-test: $\left.t_{(22)}=0.09, p=0.925\right)$

\section{DISCUSSION}

We investigated dopamine and eCB system function during effortful responding because both neuronal systems and motivation exhibit early deficits that decline across HD progression. Motivation is a process that arouses, sustains, and regulates patterns of action through coordination of sensory, motor, cognitive, and emotional functions [34]. Reduced motivation in HD-often termed apathy-is a specific symptom characterized by decreased initiation of goal-directed behaviors that is not due to gross motor, cognitive, or mood impairments [35]. This apathetic phenotype is recapitulated in HD mice as a reduced propensity to continue seeking reward during the PR task, despite no deficit in locomotion, hedonic processing of reward, or reward consumption when response costs are low [20]. In the current study, we found that this motivational deficit in adult (10 months old) HD mice is absent in younger ( 3 months old) mice. This progressive decline is characteristic of HD, as motivational impairments correlate with illness duration and disease severity [5-7]. Such deficits have previously been attributed to corticostriatal dysfunction [35], and our current and past [20] work implicates reduced dopamine release in the NAC as another contributing factor.

The NAc is conceptualized as a 'limbic-motor interface' that allows emotional information to influence basal ganglia motor circuits [36], and dopamine input to this region supports persistent responding when effortful costs are high [32]. Accordingly, the decreased magnitude of dopamine released during reward receipt in the NAC of HD mice likely influences a reduced propensity to invest effort to obtain rewards as the response ratio increases during the PR task. Moreover, elevating reward-evoked dopamine release with JZL-184 treatment is associated with reversal of this deficit. Yet, the degree to which reward-evoked dopamine release motivates future behavior or reflects prior circumstance (i.e., reward cost) is not clear. One possibility is that larger dopamine signals ascribe more value to each reward, thus promoting greater effortful investment. Additionally, JZL-184 may support a general increase in dopamine neuron activity, thus increasing tonic dopamine concentration. Both outcomes could support effortful responding as tonic dopamine levels [37] and phasic dopamine signaling [38] relate to the vigor of responding.

Dopaminergic deficits are characteristic pathogenic features of HD that arise early in asymptomatic HD mutation carriers and progressively deteriorate as symptoms worsen over the duration of illness [14]. Additionally, dopaminergic impairments are proposed to underlie dyskinetic movements that characterize motor symptoms such as chorea $[39,40]$ and cognitive decline [41], but the factors contributing to dopaminergic deficits and their contribution to apathy are not understood. Here we find that, despite a robust deficit in motivation and NAc dopamine release during the PR task, the 10-month-old HD mice exhibit intact dopamine release and uptake in a brain slice preparation, in contrast to prior measures from the more severe R6/2 mouse HD model [33]. Thus, deficits observed during the PR task likely arise from other factors such as compromised dopamine cell body firing. Because CB1 receptor signaling controls dopaminergic encoding of reward and reward seeking [18, 42], motivational and dopaminergic deficits observed in $\mathrm{HD}$ mice may arise from reduced $C B 1$ receptor signaling in the midbrain.

An impaired eCB system is a hallmark of HD [13] and is associated with behavioral deficits in HD animal models [43]. Cellular impairments include decreased CB1 receptor expression and reduced brain levels of the endogenous $C B 1$ receptor ligand 2-AG (Glass et al., 1993; [17]). 2-AG is synthesized and then released 'on-demand' predominantly from cell bodies onto presynaptic CB1 receptors following cell depolarization, elevated intracellular $\left[\mathrm{Ca}^{2+}\right]$, or certain Gq-coupled signaling cascades, and is the predominant $\mathrm{eCB}$ mediating retrograde communication in the brain [44]. Within the ventral tegmental area (VTA), 2-AG signaling facilitates dopaminergic input to the NAC and reward seeking via CB1 receptor-dependent suppression of inhibitory input onto dopamine neurons [18, 45]. Alternatively, 2-AG signaling within the NAc suppresses dopamine release and reward seeking via $\mathrm{CB} 1$ receptor-dependent suppression of excitatory input to the NAc $[29,46]$. We thus hypothesize that increased dopamine release and reward seeking observed in the current study arises from a preferential enhancement of 2-AG signaling within the VTA. In support of this hypothesis, JZL-184 does not alter dopamine released by electrical stimulation in the NAc via local actions at dopamine terminals (Supplemental fig. 7). Accordingly, the apparent second peak observed in WT mice following JZL-184 treatment (Fig. 1d) likely arises from increased midbrain dopamine cell firing prolonging the dopamine concentration change at dopamine terminals. Importantly, because systemic treatment with JZL-184 restores motivational and dopaminergic deficits in HD mice, pharmacological elevation of 2-AG levels may be a viable treatment option for apathy in HD. Notably, JZL-184 exerts similar effects in WT and HD mice, indicating that therapeutic effects of MAGL inhibition are not specific to pathogenesis in HD, but represent a general strategy for rectifying motivational and dopaminergic deficits, which are prominent in HD. Furthermore, these findings add to a growing body of work demonstrating a conserved role for $\mathrm{eCB}$ signaling in facilitating dopamine function during reward seeking $[18,42]$. 
Because the eCB system is compromised in HD, cannabinoidbased therapies have been proposed to treat HD symptoms [11, 47]. However, while targeting eCB signaling improves behavioral and neurobiological deficits in preclinical models [48, 49], clinical trials have not yielded positive results. A recent trial found no benefit of cannabis extracts that include the CB1 receptor agonist delta-9-tetrahydrocannabinol on motor, cognitive, or behavioral deficits in HD patients [50]. However, the compound used in the current study, JZL-184, amplifies ongoing eCB signaling by inhibiting the degradation of 2-AG via MAGL to indirectly increase $C B 1$ receptor binding [31]. By acting as an indirect $C B 1$ receptor agonist, MAGL inhibitors exert fewer unwanted side effects versus direct $C B 1$ receptor agonists $[51,52]$. Indeed, unlike $C B 1$ receptor agonists, JZL-184, at doses similar to those used in the current study, does not exhibit rewarding or aversive properties in place conditioning assays [53, 54]. Moreover, MAGL inhibitors are currently being investigated for treating a variety of disease states, and are in phase 1 trials for neuropathic pain and symptoms of Tourette Syndrome [55], highlighting their potential clinical viability.

Cannabinoid receptor-based treatments may be advantageous because current therapies have the potential to exacerbate psychiatric symptoms in HD such as apathy. For example, apathy is often treated with antidepressants, but-while sometimes comorbid with depression-apathy is a distinct syndrome [6] driven by unique neural mechanisms. Indeed, we have previously found that, while the Q175 HD mouse model employed in the current study exhibits diminished motivation, they do not display behaviors characteristic of depression [20]. In addition, antidepressant treatment may be problematic for apathy in HD because common antidepressants can suppress motivation by altering dopamine input to the NAC $[56,57]$. Moreover, the only FDAapproved treatment specifically for HD is TBZ, which reversibly inhibits the vesicular monoamine transporter-2 (VMAT-2) to suppress dopamine release [58]. While often effective for treating chorea, TBZ is associated with several adverse psychiatric effects $[3,59]$, and induces a prominent apathetic phenotype $[60,61]$. Thus, alternative approaches are needed for treating apathy in HD.

The current study demonstrates that $\mathrm{eCB}$ signaling controls dopaminergic encoding of reward and willingness to work for a reward. Moreover, compromised dopamine function progressively manifests as motivational deficits arise in an HD mouse model with high face and construct validity, and pharmacological enhancement of eCB signaling ameliorates these deficits. This work broadens our limited understanding of non-motor impairments in HD and informs future treatment targets for reducing disease burden.

\section{ACKNOWLEDGEMENTS}

This work was supported by a research grant from CHDI to JFC and DA041827 to DPC.

\section{ADDITIONAL INFORMATION}

Supplementary Information accompanies this paper at (https://doi.org/10.1038/ s41386-018-0107-8)

Competing interests: The authors declare no competing interests.

Publisher's note: Springer Nature remains neutral with regard to jurisdictional claims in published maps and institutional affiliations.

\section{REFERENCES}

1. MacDonald M, Ambrose C, Duyao M. A novel gene containing a trinucleotide repeat that is expanded and unstable on Huntington's disease chromosomes. Cell. 1993;72:971-83.

2. Walker FO. Huntington's disease. Lancet. 2007;369:218-28.

3. Frank S. Treatment of Huntington's disease. Neurotherapeutics. 2014;11:153-60.
4. Epping EA, Kim Jl, Brashers-Krug TM, Anderson KE, McCusker E, Luther J, et al. Longitudinal psychiatric symptoms in prodromal Huntington's disease: a decade of data. Am J Psychiatry. 2016;173:184-92.

5. Paulsen JS, Langbehn DR, Stout JC, Aylward E, Ross CA, Nance M, et al. Detection of Huntington's disease decades before diagnosis: The Predict-HD study. J Neurol Neurosurg Psychiatry. 2008;79:874-80.

6. Naarding $P$, Janzing JG, Eling $P$, van der Werf $S$, Kremer $B$. Apathy is not depression in Huntington's disease. J Neuropsychiatry Clin Neurosci. 2009;21:266-70.

7. Thompson JC, Harris J, Sollom AC, Stopford CL, Howard E, Snowden JS, et al. Longitudinal evaluation of neuropsychiatric symptoms in Huntington's disease. J Neuropsychiatry Clin Neurosci. 2012;24:53-60.

8. Reiner A, Albin RL, Anderson KD, D'Amato CJ, Penney JB, Young AB. Differential loss of striatal projection neurons in Huntington disease. Proc Natl Acad Sci USA. 1988;85:5733-7.

9. Vonsattel JP, DiFiglia M. Huntington disease. J Neuropathol Exp Neurol. 1998;57:369-84.

10. Wilson H, De Micco R, Niccolini F, Politis M. Molecular imaging markers to track Huntington's disease pathology. Front Neurol. 2017;8:11.

11. Garcia C, Palomo-Garo C, Gomez-Galvez Y, Fernandez-Ruiz J. Cannabinoiddopamine interactions in the physiology and physiopathology of the basal ganglia. Br J Pharmacol. 2016;173:2069-79.

12. Cepeda C, Murphy KP, Parent M, Levine MS. The role of dopamine in Huntington's disease. Prog Brain Res. 2014;211:235-54.

13. Pazos MR, Sagredo O, Fernandez-Ruiz J. The endocannabinoid system in Huntington's disease. Curr Pharm Des. 2008;14:2317-25.

14. Richfield EK, O'Brien CF, Eskin T, Shoulson I. Heterogeneous dopamine receptor changes in early and late Huntington's disease. Neurosci Lett. 1991;132:121-6.

15. Glass M, Dragunow M, Faull RL. The pattern of neurodegeneration in Huntington's disease: a comparative study of cannabinoid, dopamine, adenosine and $\mathrm{GABA}(\mathrm{A})$ receptor alterations in the human basal ganglia in Huntington's disease. Neuroscience. 2000;97:505-19.

16. Kish SJ, Shannak K, Hornykiewicz O. Elevated serotonin and reduced dopamine in subregionally divided Huntington's disease striatum. Ann Neurol. 1987;22:386-9.

17. Dowie MJ, Bradshaw HB, Howard ML, Nicholson LF, Faull RL, Hannan AJ, et al. Altered $\mathrm{CB} 1$ receptor and endocannabinoid levels precede motor symptom onset in a transgenic mouse model of Huntington's disease. Neuroscience. 2009;163:456-65.

18. Oleson EB, Beckert MV, Morra JT, Lansink CS, Cachope R, Abdullah RA, et al. Endocannabinoids shape accumbal encoding of cue-motivated behavior via CB1 receptor activation in the ventral tegmentum. Neuron. 2012;73:360-73.

19. Covey DP, Mateo Y, Sulzer D, Cheer JF, Lovinger DM. Endocannabinoid modulation of dopamine neurotransmission. Neuropharmacology. 2017;124: 52-61.

20. Covey DP, Bunner KD, Schuweiler DR, Cheer JF, Garris PA. Amphetamine elevates nucleus accumbens dopamine via an action potential-dependent mechanism that is modulated by endocannabinoids. Eur J Neurosci. 2016:43:1661-73.

21. Menalled LB, Kudwa AE, Miller S, Fitzpatrick J, Watson-Johnson J, Keating N, et al. Comprehensive behavioral and molecular characterization of a new knock-in mouse model of Huntington's disease: ZQ175. PLoS One. 2012;7:e49838.

22. Clark JJ, Sandberg SG, Wanat MJ, Gan JO, Horne EA, Hart AS, et al. Chronic microsensors for longitudinal, subsecond dopamine detection in behaving animals. Nat Methods. 2010;7:126-9.

23. Heien ML, Khan AS, Ariansesn JL, Cheer JC, Phillips PE, Wassum KM, et al. Realtime measurement of dopamine fluctuations after cocaine in the brain of behaving rats. Proc Natl Acad Sci USA. 2005;102:10023-8.

24. Keithley RB, Wightman RM. Assessing principal component regression prediction of neurochemicals detected with fast-scan cyclic voltammetry. ACS Chem Neurosci. 2011;2:514-25

25. Roberts JG, Toups JV, Eyualem E, McCarty GS, Sombers LA. In situ electrode calibration strategy for voltammetric measurements in vivo. Anal Chem. 2013;85:11568-75.

26. John $C E$, Jones $S R$. Exocytotic release of dopamine in ventral tegmental area slices from C57BL/6 and dopamine transporter knockout mice. Neurochem Int. 2006;49:737-45.

27. Yorgason JT, Espana RA, Jones SR. Demon voltammetry and analysis software: analysis of cocaine-induced alteration in dopamine signaling using multiple kinetic measures. J Neurosci Methods. 2011;202:158-64.

28. Hodos W. Progressive ratio as a measure of reward strength. Science. $1961 ; 134: 943-4$

29. Mateo Y, Johnson KA, Covey DP, Atwod BK, Wang HL, Zhang S, et al. Endocannabinoids on cortical terminals orchestrate local modulation of dopamine release in the nucleus accumbens. Neuron. 2017;96:1112-26.

30. Oleson EB, Cachope R, Fitoussi A, Tsutsui K, Wu S, Gallegos JA, et al. Cannabinoid receptor activation shifts temporally engendered patterns of dopamine release. Neuropsychopharmacology. 2014;39:1441-52. 
31. Long JZ, Li W, Booker L, Burston JJ, Kinsey SG, Schlosburg JE, et al. Selective blockade of 2-arachidonoylglycerol hydrolysis produces cannabinoid behavioral effects. Nat Chem Biol. 2009;5:37-44.

32. Salamone JD, Pardo M, Yohn SE, Lopez-Cruz L, SanMiguel N, Correa M. Mesolimbic dopamine and the regulation of motivated behavior. Curr Top Behav Neurosci. 2016;27:231-57.

33. Johnson MA, Rajan V, Miller CE, Wightman RM. Dopamine release is severely compromised in the R6/2 mouse model of Huntington's disease. J Neurochem. 2006;97:737-46.

34. Salamone JD. Complex motor and sensorimotor functions of striatal and accumbens dopamine: involvement in instrumental behavior processes. Psychopharmacology (Berl). 1992;107:160-74.

35. Levy R, Dubois B. Apathy and the functional anatomy of the prefrontal cortexbasal ganglia circuits. Cereb Cortex. 2006;16:916-28.

36. Mogenson GJ, Jones DL, Yim CY. From motivation to action: functional interface between the limbic system and the motor system. Prog Neurobiol. 1980;14:69-97.

37. Niv Y. Cost, benefit, tonic, phasic: what do response rates tell us about dopamine and motivation? Ann NY Acad Sci. 2007;1104:257-376.

38. Ko D, Wanat MJ. Phasic dopamine transmission reflects initiation vigor and exerted effort in an action- and region-specific manner. J Neruosci. 2016;36:2202-11.

39. Bird ED. Chemical pathology of Huntington's disease. Annu Rev Pharmacol Toxicol. 1980;20:533-51.

40. Klawans HC, Paulson GW, Barbeau A. Predictive test for Huntington's chorea. Lancet. 1970;2:1185-6.

41. Backman L, Robins-Wahlin TB, Lundin A, Ginovart N, Farde L. Cognitive deficits in Huntington's disease are predicted by dopaminergic PET markers and brain volumes. Brain. 1997;120(Pt 12):2207-17.

42. Covey DP, Dantrassy HM, Zlebnik NE, Gildish I, Cheer JF. Compromised dopaminergic encoding of reward accompanying suppressed willingness to overcome high effort costs is a prominent prodromal characteristic of the Q175 mouse model of Huntington's disease. J Neurosci. 2016;36:4993-5002.

43. Blàzquez C, Chiarlone A, Sagredo O, Aguado T, Pazos MR, Resel E, et al. Loss of striatal type 1 cannabinoid receptors is a key pathogenic factor in Huntington's disease. Brain. 2011;134:119-36.

44. Ohno-Shosaku T, Kano M. Endocannabinoid-mediated retrograde modulation of synaptic transmission. Curr Opin Neurobiol. 2014;29:1-8.

45. Wang H, Treadway T, Covey DP, Cheer JF, Lupica CR. Cocaine-induced endocannabinoid mobilization in the ventral tegmental area. Cell Rep. 2015;12:1997-2008

46. Sidlo $\mathrm{Z}$, Reggio $\mathrm{PH}$, Rice ME. Inhibition of striatal dopamine release by $\mathrm{CB} 1$ receptor activation requires nonsynaptic communication involving $\mathrm{GABA}, \mathrm{H} 2 \mathrm{O} 2$, and KATP channels. Neurochem Int. 2008;52:80-8.

47. Fernandez-Ruiz J, Hernandez M, Ramos JA. Cannabinoid-dopamine interaction in the pathophysiology and treatment of CNS disorders. CNS Neurosci Ther 2010;16:e72-91.
48. Lastres-Becker I, Hansen HH, Berrendero F, De Miguel R, Pèrez-Rosado A, Manzanares $\mathrm{J}$, et al. Alleviation of motor hyperactivity and neurochemical deficits by endocannabinoid uptake inhibition in a rat model of Huntington's disease. Synapse. 2002;44:23-35.

49. Valdeolivas S, Sagredo O, Delgado M, Pozo MA, Fernandez-Ruiz J. Effects of a Sativex-like combination of phytocannabinoids on disease progression in R6/2 mice, an experimental model of Huntington's disease. Int J Mol Sci. 2017;18:E684.

50. Lòpez-Sendòn Moreno JL, Garcia Caldentey J, Trigo Cubillo P, Ruiz-Romero C, Garcia Ribas G, Alonso Arias MA, et al. A double-blind, randomized, cross-over, placebo- controlled, pilot trial with Sativex in Huntington's disease. J Neurol. 2016:263:1390-1400

51. Grabner GF, Zimmermann R, Schicho R, Taschler U. Monoglyceride lipase as a drug target: at the crossroads of arachidonic acid metabolism and endocannabinoid signaling. Pharmacol Ther. 2017;175:35-46.

52. Mulvihill MM, Nomura DK. Therapeutic potential of monoacylglycerol lipase inhibitors. Life Sci. 2013;92:492-7.

53. Gamage TF, Ignatowska-Jankowska BM, Muldoon PP, Cravatt BF, Damaj MI Lichtman AH. Differential effects of endocannabinoid catabolic inhibitors on morphine withdrawal in mice. Drug Alcohol Depend. 2015;146:7-16.

54. Muldoon PP, Chen J, Harenza JL, Abdullah RA, Sim-Selley LJ, Cravatt BF, Miles MF, Chen X, Lichtman AH, Damaj MI. Inhibition of monoacylglycerol lipase reduces nicotine withdrawal. Brit J Pharmacol. 2015;172:869-82.

55. Granchi C, Caligiuri I, Minutolo F, Rizzolio F, Tuccinardi T. A patent review of monoacylglycerol lipase (MAGL) inhibitors (2013-7). Expert Opin Ther Pat. 2017;27:1341-51.

56. Hoehn-Saric R, Lipsey JR, McLeod DR. Apathy and indifference in patients on fluvoxamine and fluoxetine. J Clin Psychopharmacol. 1990;10:343-5.

57. Yohn SE, Collins SL, Contreras-Mora HM, Errante EL, Rowland MA, Correa M, et al. Not all antidepressants are created equal: differential effects of monoamine uptake inhibitors on effort-related choice behavior. Neuropsychopharmacology. 2016:41:686-94.

58. Paleacu D. Tetrabenazine in the treatment of Huntington's disease. Neu ropsychiatr Dis Treat. 2007;3:545-51.

59. Killoran A, Biglan KM. Current therapeutic options for Huntington's disease: good clinical practice versus evidence-based approaches? Mov Disord. 2014;29: 1404-13.

60. Randall PA, Lee CA, Nunes EJ, Yohn SE, Nowak V, Khan B, et al. The VMAT-2 inhibitor tetrabenazine affects effort-related decision making in a progressive ratio/chow feeding choice task: reversal with antidepressant drugs. PLoS One. 2014;9:e99320.

61. Yohn SE, Thompson C, Randall PA, Lee CA, Müller CE, Bagi Y, et al. The VMAT-2 inhibitor tetrabenazine alters effort-related decision making as measured by the T-maze barrier choice task: reversal with the adenosine A2A antagonist MSX-3 and the catecholamine uptake blocker bupropion. Psychopharmacology (Berl). 2015;232:1313-23. 